\title{
Effect of Saudi and Egyptian pomegranate polyphenols in regulating the activity of PON1, PON2 and lipid profile for preventing coronary heart disease
}

\author{
Mona Nasser BinMowyna ${ }^{1}$ Manal Abdulaziz Binobead ${ }^{2}$, Nawal Abdullah Al Badr², Sahar \\ Abdulaziz AlSedairy ${ }^{2}$, Islam Abdul Rahim Elredh ${ }^{3}$, Wedad Saeed Al-Qahtani ${ }^{4}$
}

${ }^{1}$ Department of Food science, College of education, Shaqra University, Riyadh, Saudi Arabia

${ }^{2}$ College of Food Science \& Agriculture, Department of Food Science \& Nutrition, King Saud University, Riyadh, Saudi Arabia.

${ }^{3}$ College of Food Science \& Agriculture, Veterinarian (Supervisor helper of animal house), King Saud University, Riyadh, Saudi Arabia.

${ }^{4}$ College of Science, King Saud University, Riyadh, Saudi Arabia.

\section{Corresponding Author;}

Wedad Saeed Alqahtani

Naif Arab University for Security Sciences, College of Forensic Sciences, Forensic Biology Department

Email;

dr.wedad.alqahtani@nauss.edu.sa

436203668@student.ksu.edu.sa 


\section{Abstract}

Saudi and Egyptian pomegranate juice (APJ and EPJ) contains potent polyphenols antioxidants which were found to reduce serum and macrophage oxidative stress. The protective effects of APJ and EPJ against atherogenicity were evaluated by feeding mice with hypercholesterolemic diet supplemented with PJ. PJ consumption reduced mice serum Triglycerides (TG), Total cholesterol (TC) and (low density lipoprotein (LDL-c) and increase in the High-density lipoprotein (HDL-c) compared with mouse with control diet or atherogenic diet. The risk ratio and atherogenic index were significantly lower in either APJ or EPJ supplemented group. Paraoxonase 1 (PON1) which remains associated with HDL showed significant increase in the activity in mice supplemented with PJ compared to mice with atherogenic diet (AD). PJ consumption significantly reduced lipid peroxidation and increased glutathione levels. The reduction in lipid peroxidation varied from 57 to $76 \%$. PJ supplementation reduced peritoneal macrophages (MPM) mediated oxidation of LDL by 68 to $82 \%$ and decreased mouse peritoneal macrophages (MPM) Ox-LDL uptake by 31 to $48 \%$. A significant up regulation of macrophage PON2 activity was induced by PJ reducing the macrophage oxidative stress. In conclusion, PJ consumption resulted in anti-oxidative and antiatherogenic effects on serum and macrophages which could prevent atherosclerosis and coronary heart diseases.

Key words: Pomegranate polyphenols, Paraoxonase activity, Serum cholesterol, Atherogenic diet 


\section{Introduction}

Over the last few years, the role of polyphenols in reducing risk of chronic diseases has received much attention. Polyphenols are naturally occurring phytochemicals contained in most of the fruits, vegetables, cereals and beverages (Grosso, 2018). Recent evidences suggested potential beneficial effects of polyphenols in regulating vascular and endothelial function by lowering blood pressure, improving endothelial function, increasing antioxidant and antiinflammatory activities, inhibiting low-density lipoprotein oxidation, and reducing inflammatory responses (Giglio et al., 2018). They are associated with prevention and treatment of cardiovascular diseases (CVDs) reducing CVD-related mortality. One of the most evident biomarkers for CVD risk is high LDL (Low-density lipoprotein) cholesterol and low HDL (High-density lipoprotein) cholesterol (Hollands et al., 2018). Atherosclerosis the major cause of CVD is mediated by endothelial dysfunction caused due to accumulation of LDL. Endothelial dysfunction leads to infiltration of LDL and their subsequent oxidation to oxidized-LDL (oxLDL) by interacting with superoxide anions (Moss et al., 2018). High oxidative stress in conditions such as CVD, reduces the serum concentration and activity of paraoxonase-1 (PON1) (Dizaji et al., 2018). Polyphenols reduces atherosclerosis by increasing HDL, reducing LDL and total cholesterol (Santhakumar et al., 2018).

Pomegranate (Punica granatum L.) has traditionally been used for treatment of many chronic diseases due to its multi-factorial beneficial effects. Pomegranate extracts are known to have anti-inflammatory, antidiabetic and antioxidant activity (AlMatar, 2018). Studies revealed that pomegranate is rich in polyphenols and includes anthocyanins and anthoxanthins. Ellagic acid and hydrolysable ellagitannins are the principal polyphenols component having antioxidant properties. Punicalagin, an ellagitannins found in pomegranate is known for its antiatherogenic activities (Les et al., 2018). It prevents accumulation of lipids in macrophages and foam cell formation (Atrahimovich et al., 2018). Other hydrolysable tannins include punicalain and gallic acid (Rosenblat et al., 2015).

Paraoxonase is an HDL-associated esterase that prevents oxidation of lipids in lipoproteins. Studies revealed that consumption of pomegranate juice (PJ) promotes binding of HDL to paraoxonase 1 (PON1), thereby increasing the hydrolytic and peroxidative activity of paraoxonase (Kaplan, 2001). PON1 is synthesized in the liver and transported in plasma in 
association with HDL. The decrease in the PON1 activity may lead to higher levels of cholesterol (Estrada-Luna et al., 2018). Other associated paraoxonase are PON2 and PON3. PON2 is expressed in most tissues including macrophages and plays a crucial role in preventing atherosclerosis. PON3 is found associated with HDL, mitochondria and endoplasmic reticulum (Moya and Manez, 2018). Studies suggested that pomegranate polyphenols reduce macrophage oxidative stress and formation of foam cells by over expression of macrophage PON2. PON2 prevent macrophage oxidation and oxidation of LDL by inhibiting the formation and release of reactive oxygen species (ROS) and reactive nitrogen species (RNS) (Aviram and Rosenblat,, 2012).

The present study envisaged the effect of polyphenols of Saudi and Egyptian Pomegranate juice in regulating the activity of PON1, PON2, serum lipids and macrophage oxidative stress, and their role in preventing the coronary heart disease in mice.

\section{Materials and methods}

\section{Pomegranate fruits}

Ripened fruits of the Saudi pomegranate were obtained from the local market of Taif and Egyptian pomegranate fruits from the local market of Riyadh city. The entire fruit was squeezed with a juicer and filtered through $15 \mu \mathrm{m}$ membrane to remove insoluble residue. Fresh PJ was prepared daily during the entire experimental period.

\section{Experimental animals}

Twenty-five CD1 mice were obtained from the experimental animal's center, King Saud University. Mice weighing $25 \pm 5 \mathrm{~g}$ were used in the study. The mice were housed in wirebottomed cages and maintained in controlled environment (temperature of $22 \pm 2{ }^{\circ} \mathrm{C}$, relative humidity of $50 \pm 5 \%$, and $12 \mathrm{~h}$ light/dark cycle). Mice were divided into five groups, comprised of five mice per group. The first group was fed with normal control diet (CD), the second group with high-fat diet referred as atherogenic diet (AD), the third group was fed with high-fat diet supplemented with $200 \mu \mathrm{L}$ Saudi pomegranate juice (APJ) containing $0.42 \mu \mathrm{mol}$ of total polyphenols, the fourth groups was fed with high-fed diet supplemented with $200 \mu \mathrm{L}$ Egyptian pomegranate juice (EPJ) containing $0.39 \mu \mathrm{mol}$ of total polyphenols, the fifth group was fed with high-fat diet supplemented with $200 \mu \mathrm{L}$ mixture of both APJ and EPJ. Supplementation was carried out for six month and diets was delivered directly to the stomach using oral gavage. 


\section{Diets used in the experiment}

The high-fat atherogenic diet contained (w/w) saturated fat $20 \%$, cholesterol $1.5 \%$, mineral salts mixture $2.5 \%$, casein $20 \%$, vitamin mixture $1 \%$, cellulose $5 \%$ and starch $50 \%$. The control group received water without PJ, saturated fat, cholesterol and casein. All the groups after supplementation had access to drinking water of 2-5 mL/day. Diets were stored at $4^{\circ} \mathrm{C}$ in plastic containers until used.

\section{Serum analyzes}

At the end of the experiment, the mice were grafted and anesthetized using diethyl ether. The blood samples were then withdrawn and separated using a centrifuge. Serum analyses of total cholesterol, triglyceride, HDL-c, LDL-c were determined as previously described (Rosenblat et al., 2015).

\section{Serum lipid peroxidation}

The extent of lipid peroxidation in plasma was determined by measurement of monodialdehyde (MDA) formation at $534 \mathrm{~mm}$ using the thiobarbituric acid reactive substances (TBARS) method. The reduced glutathione (GSH) in the plasma was estimated by its reaction with dithio-bis-2-nitrobenzoic acid (DTNB) that give a yellow coloured complex with maximum absorption at $412 \mathrm{~nm}$.

\section{Serum PON1 activity}

Serum PON1 activity was measured using phenyl acetate as the substrate (Gaidukov and Tawfik, 2005). Initial rates of substrate hydrolysis were determined spectrophotometrically at $270 \mathrm{~nm}$. The assay mixture included $5 \mu \mathrm{L}$ serum (diluted 1:3), $1.0 \mathrm{mmol} / \mathrm{L}$ phenylacetate, and 1 $\mathrm{mmol} / \mathrm{L} \mathrm{CaCl}_{2}$ in $50 \mathrm{mmol} / \mathrm{L}$ Tris HCL ( $\mathrm{pH} \mathrm{8.0).} \mathrm{The} \mathrm{results} \mathrm{were} \mathrm{calculated} \mathrm{assuming} \mathrm{the} \mathrm{molar}$ extinction coefficient of phenyl acetate to be $1,310 \mathrm{~L} \mathrm{~mole} \mathrm{~cm}^{-1}$. One unit of arylesterase activity is equal to $1 \mu \mathrm{moL}$ of phenylacetate hydrolyzed $/ \mathrm{min} / \mathrm{mL}$.

\section{Mouse peritoneal macrophages (MPM)}

MPM were harvested from the peritoneal fluid of the mice, 3 days after IP injection into each mouse of $3 \mathrm{~mL}$ of aged thioglycolate $(20 \mathrm{~g} / \mathrm{L})$ in saline. The cells $\left(10-20 \times 10^{6} / \mathrm{mouse}\right)$ were washed and centrifuged three times with phosphate-buffered saline (PBS) at $1000 \mathrm{x} \mathrm{g}$ for $10 \mathrm{~min}$. 


\section{MPM mediated oxidation of $L D L$}

LDL was separated from plasma by density-gradient ultracentrigugation. MPN were incubated with LDL $(100 \mu \mathrm{g}$ of protein $/ \mathrm{mL})$ in the presence of $5 \mu \mathrm{M}$ of $\mathrm{CuSo}_{4}$ for $5 \mathrm{~h}$ at $37^{\circ} \mathrm{C}$. LDL oxidation was measured by TBARS assay (Buege and Aust, 1978).

\section{MPM uptake of $O x-L D L$}

Ox-LDL was conjugated to fluoroisothiocyanate (FITC) for cellular lipoprotein uptake studies (Bass et al., 1983). MPM were incubated at $37^{\circ} \mathrm{C}$ for $3 \mathrm{~h}$ with FITC-conjugated Ox-LDL at a final concentration of $25 \mu \mathrm{g}$ of protein $/ \mathrm{mL}$. The uptake of the lipoprotein was determined by flow cytometry. Cellular fluorescence was measured in terms of mean fluorescence intensity (MFI).

\section{MPM PON2 activity}

Lactonase activity was measured in intact cells using dihydrocumarin (DHC) as a substrate. DHC $(1 \mathrm{mmoL} / \mathrm{L})$ was added to the cells in $1 \mathrm{~mL}$ of $1 \mathrm{mmol} / \mathrm{L} \mathrm{CaCl}_{2}$ in $50 \mathrm{mmol} / \mathrm{L}$ of Tris HCL, $\mathrm{pH}$ 8.0. One unit of lactonase activity $=1 \mu \mathrm{moL}$ of $\mathrm{DHC}$ hydrolyzed $/ \mathrm{min}$. The absorbance was monitored at 270nm for 10 min after substrate addition.

\section{Statistical Analysis}

Data obtained from the respective experiments were pooled and the means from these were used in statistical analysis using SPSS version-25. P values $<0.01$ and $<0.05$ were considered as significant. Means were separated with Duncan Multiple Range Test (DMRT) in respective tables and further verified using Akaike's information criterion (AIC) and Hurvich and Tsai's criterion. 


\section{Results and Discussions}

The effect of hypercholesterolemic diets on different treated groups revealed that body weight gain significantly reduced in the group supplemented with APJ and EPJ compared to mice group with either control diet (CD) and atherogenic diet (AD). The reduction in net weight gain varies from $20.6 \mathrm{~g}$ to $22.4 \mathrm{~g}$ in APJ and EPJ supplemented diet (Fig.1). The result is in consistent with the earlier findings on reduction of body weight of mice treated with pomegranate seed oil and seed residue (Elbandy and Ashoush, 2012). However, more reduction in body weight was noticed in group treated with both APJ and EPJ supplemented diet (7.6 g). The result thus revealed that the dual effect causes more reduction in body weight compared to individual treatment with either APJ or EPJ alone. The least information criteria of net body weight gain according to Akaike's information criterion (AIC) and Hurvich and Tsai's criterion (AICC) was $812.851\left(R^{2}=0.42\right)$. Similarly, significant reductions in the relative heart weight were observed in the treated groups compared to control. The reduction in weight is $80.7 \%$ and $69.8 \%$ in APJ and EPJ supplemented mice group and 67.5\% in APJ+EPJ supplemented mice group compared to $\mathrm{AD}$ group.

The plasma lipid profile revealed significant higher level of TG (172.12 mg/dl), TC $(153.34 \mathrm{mg} / \mathrm{dl})$ and LDL-c $(72.39 \mathrm{mg} / \mathrm{dl})$ and lower level of HDL-c (33. 45mg.dl) in mice supplemented with atherogenic diet (AD). While mice supplemented with APJ, EPJ and combined APJ and EPJ diet showed significant lower level of TG, TC, and LDL-c and higher level of HDL-c compared to AD supplemented group (Fig.2). Thus, the present findings revealed decrease in the lipid fractions in PJ supplemented mice group, thereby reducing atherosclerotic process and chance of cardiovascular diseases (Berek and Bobinski, 2009). High HDL-c signifies anti-atherogenic properties and inhibition of LDL-oxidation. Similarly, the risk ratio (RR) and atherogenic index (AI) values the indicator of coronary and cardiovascular risk were significantly lower in mice supplemented with either APJ or EPJ or APJ and EPJ combined supplemented diets.

The present result revealed significant reduction in lipid peroxidation (MDA) in mice treated with either APJ or EPJ compared to mice treated with control diet (CD) and atherogenic diet (AD). The reduction varies from 57.1 to $76.3 \%$ in EPJ and APJ supplemented group (Fig.3). Thus, this is a clear indicative of effect of pomegranate juice in reduction of autocatalytic 
activities of lipid peroxidation thereby minimizing tissue damage. The reduction is more pronounced in group treated with both APJ and EPJ. Similarly, glutathione (GSH) and indicator of antioxidant activity, significantly increased in mice treated with APJ (47.2\%) and EPJ (43.4\%) as well as in combined application of APJ and EPJ (44.2\%). In contrast, the level is significantly higher in mice with atherogenic diet. The reduction in MDA and enhancement of GSH level indicated that both APJ and EPJ have antioxidant properties capable of preventing tissue damage or cell death $\left(p<0.05, R^{2}=0.59\right)$.

Serum paraoxonase (PON1) enzyme activity significantly decreased in mice supplemented with hypercholesterolemic diet compared to control and PJ treated (APJ, EPJ and APJ+EPJ) mice groups (Fig.4). The present result indicated that high-fat diet effects the paraoxonase activity increasing the risk of atherosclerosis resulting in coronary diseases (Estrada-Luna et al., 2018). There was marked increase in the paraoxonase activity in the mice group treated with PJ diet. The serum paraoxonase activity in PJ treated mice was 58\% higher that mice with mice with atherogenic diet. This result is an indicative of high PON1 activity in PJ treated mice protecting LDL and HDL against oxidative processes, preventing the formation of atherogenic ox-LDL molecules (Zielaskowska and Olszewska, 2006). Research indicates that serum PON1 activity is inversely proportional to risk of atherosclerosis which confirms its protective properties in preventing cardiovascular diseases (Rosenblat et al., 2006).

\section{MPM mediated oxidation of $L D L$}

APJ, EPJ or APJ+EPJ supplemented mice groups showed significant decrement by 82.0 and 79.8\%, 78.6 and 76.1\%, 70.3 and 68.3\% respectively in MPM mediated LDL oxidation compared to $\mathrm{CD}$ and $\mathrm{AD}$ supplemented mice groups (Fig.5). The result is in conformity with some of the earlier findings (Aviram and Rosenblat, 2004; Rosenblat et al., 2010). The result indicated that APJ was the most potent antioxidant against LDL oxidation. The decrement is significant with different PJ treatment as evidenced by correlation coefficient of $R^{2}=0.88$, $(p<0.05)$.

\section{MPM uptake of $O x-L D L$}

As evidenced from earlier studies oxidized LDL uptake by macrophages can lead to accumulation of macrophage cholesterol and formation of foam cell (Aviram and Rosenblat, 2004). In the present study, MPM from PJ treated mice group showed decreasing trend in Ox- 
LDL uptake compared to control and atherogenic group (Fig.6). The uptake was significantly decreased by $47.8 \%$ in MPM from APJ supplemented group, 46.5\% from EPJ supplemented group and 36.7\% from APJ + EPJ supplemented group. Similar result was obtained in some of the earlier studies (Rom et al., 2016).

MPM PON2 lactonase activity significantly increased by $54.6 \%, 50 \%$ and $48 \%$ in APJ, EPJ and APJ+EPJ supplemented mice group compared to group with atherogenic diet (Fig.7). The increase is significant with different PJ treatment as evidenced by correlation coefficient of $R^{2}=0.54,(p<0.05)$. The finding is pertinent to some of the earlier studies in which PJ was shown to increase in macrophage PON2 activity (Rosenblat et al., 2010). The increase is 2 to 3 fold when compared to group with control diet. This increase may be attributed to increased PON2 relative gene expression following PJ supplementation. The present result indicates that PON2 may act as cellular antioxidant reducing oxidative stress through its anti-atherogenic role (Aviram and Rosenblat, 2004).

\section{Conclusion}

The present study reveals that dietry Saudi and Egyptian pomegranate juice significantly reduces oxidative stress by upregulating PON1 and PON2 activity and prevents LDL oxidation thus proofing its protective role in the progression of coronary heart diseases. The findings indicate that pomegranate juices can be preferred as a potential nutritional therapy in prevention of atherosclerosis and thereby overcoming the possible risk of developing heart diseases. This study highlighted the potential antioxidant effect of pomegranate polyphenols. 


\section{References}

AlMatar, M., Islam, M.R., Albarri, O., Var I. and Koksal, F. (2018). Pomegranate as a possible treatment in reducing risk of developning wound healing, obesity, neurodegenerative disorders and diabetes mellitus. Mini-review in Medicinal Chemistry. 18(6): 507526.

Atrahimovich, D., Samson, A.O., Khattib, A., Vaya, J. and Khatib, S. (2018). Punicalagin decreases serum glucose levels and increases PON1 activity and HDL antiinflammatory values in Balb/c mice fed a high-fat diet. Oxidative Medicines and Cellular Longevity. Article ID 2673076, 8 pages, https://doi.org/10.1155/2018/2673076.

Aviram, M. and Rosenblat, M. (2004). Paraoxonases 1, 2 and 3 oxidaative stress and macrophage foam cell formation during atherosclerosis development. Free Radical Biology and Medicine. 37(9):1304-1316.

Aviram, M. and Rosenblat, M. (2012). Pomegranate protection against cardiovascular diseases. Evidence-Based Complementary and Alternative Medicine. ID382763, doi:10.1155/2012/382763.

Berek, K. and Bobinski R. (2009). Atherosclerosis-a multifactorial disease. Problemy Pielegniarstwa. 17:257-262.

Buege, J.A. and Aust, S.D. (1978). Microsomal lipid peroxidation. Methods Enzymol. 52:30210.

Dijaji, B.F., Rivandi, M., Javandoost, A, Karimian, M.S., Raei, A., Sahebkar, A., Ferns, G., Mobarhan, M.G. and Pasdar, A. (2018). Association of genetic polymorphism of PON1 and CTEP with the presence of metabolic syndrome; the effects of genotypes on their serum activity and concentrations. The Egyptian Journal of Medical Human Genetics. 19:43-48.

Elbandy, M.M. and Ashoush, I.S. (2012). Phytochemicals in pomegranate seeds and their effect as hypolipidemic agent in hypercholesterolemic mice. World journal of Diary and Food Sciences. (7(1):85-92.

Estrada-Luna, D., Martinez-Hinojosa, E., Cancio-Diaz, J.C., Belefant-Miller, H., LopezRodriguez, G. and Betanzos-Cabrera, G. (2018). Daily supplementation with fresh 
pomegranate juice increases paraoxonase 1 expression and activity in mice fed a high-fat diet. European Journal of Nutrition. 57(1):383-389.

Gaidukov, L. and Tawfik, D.S.(2005). High affinity, stability, and lactonase activity of serum paraoxonase PON1 anchored on HDL with ApoA-I. Biochemistry. 44:11842- 11854.

Giglo, R.V., Patti, A.M., Cicero, A.F.G., Lippi, G., Rizzo, M., Toth, P.P., Banach, M. (2018). Polyphenols: Potential use in the prevention and treatment of cardiovascular diseases. Current Pharmaceutical Design. 24(2): 239-258.

Grosso, G. (2018). Effects of polyphenols-rich foods on human health. Nutrients. 10(8): 1089, doi:10.3390/nu10081089.

Hollands, W.J., Armah, C.N., Doleman, J.F., Perez-Moral, N., Winterbone, M.S. and Kroon, P.A. (2018). 4-week consumption of anthocyanin-rich blood orange juice does not affect LDL-cholesterol of other biomarkers of CVD risk and glycemia compared with orange juice: a randomized controlled trial. British Journal of Nutrition. 119: 415-421.

Kaplan, M., Hayek T., Raz, A., Coleman, R., Dornfeld, L., Vaya, J. and Aviram, M. (2001). Pomegranate juice supplementation to atherosclerotic mice reduces macrophage lipid peroxidation, cellular cholesterol accumulation and development of atherosclerosis. The Journal of Nutrition.131(8): 2082-2089.

Les, F., Arbones-Mainar, J.M., Valero, M.S. and Lopez, V. (2018). Pomegranate polyphenols and urolithin A inhibit $\alpha$-glucosidase, dipeptidyl peptidase-4, lipase, triglyceride accumulation and adipogenesis related genes in 3T3-L1 adipocyte-like cells. Journal of Ethnopharmacology. 220:67-74.

Moss, J.W.E., Williams, J.O. and Ramji, D.P. (2018). Nutraceuticals as therapeutic agents for atherosclerosis. Biochemica et Biophysica-Molecular Basis of Disease. 1864 (5): 1562-1572.

Moya, C. and Manez, S. (2018). Paraoxonases: metabolic role and pharmacological projection. Naunyn-Schmiedeberg's Archives of Pharmacology. 391(4): 349-359.

Rom, O., Korach-Rechtman, H., Hayek, T., Damin-Poleg, Y., Bar, H., Kashi, Y. and Aviram, M. (2016). Acrolein increases macrophage atherogenicity in association with gut microbiota remodeling in atherosclerotic mice: protective role for the polyphenolsrich pomegranate juice. Arch. Tocicol. DOI 10.1007/s00204-016-1859-8. 
Rosenblat, M., Gaidukov, L., Khersonsky, O., Vaya, J., Oren, R., Tawfik, D.S. and Aviram, M. (2006). The catalytic histidine dyad of high density lipoprotein associated serum paraoxonase-1 (PON1) is essential for PON1-mediated inhibition of low density lipoprotein oxidation and stimulation of macrophage cholesterol efflux. J. Biol. Chem. 281:7657-7665.

Rosenblat, M., Volkova, N. and Aviram, M. (2010). Pomegranate juice (Pj) consumption antioxidative properties on mouse macrophages, but not $\mathrm{Pj}$ beneficial effects on macrophage cholesterol and triglyceride metabolism, are mediated via $\mathrm{Pj}$-induced stimulation of macrophage PON2. Athrrosclerosis. 212:86-92.

Rosenblat, M., Volkova, N., Borochov-Neori, H., Judeinstein, S. and Aviram, M. (2015). Antiatherogenic properties of date vs. pomegranate polyphenols: the benefits of the combination. Food and Function. 6(5):1496-1509.

Santhakumar, A.B., Battino, M. and Alvarez-Suarez, J.M. (2018). Dietry polyphenols: Structures, bioavailability and protective effects against atherosclerosis. Food and Chemical Toxicology. 113:49-65.

Zielaskowska, J. and Olszewska-Słonina, D. (2006). The polymorphism of paraoxonase and its effects in physiological and pathological processes. Adv. Clin. Exp. Med. 15:10731078 . 


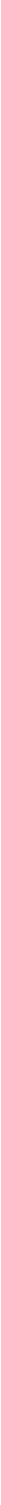

Fig.1 Variation in body weight and relative heart weight in high- 
LDL-C

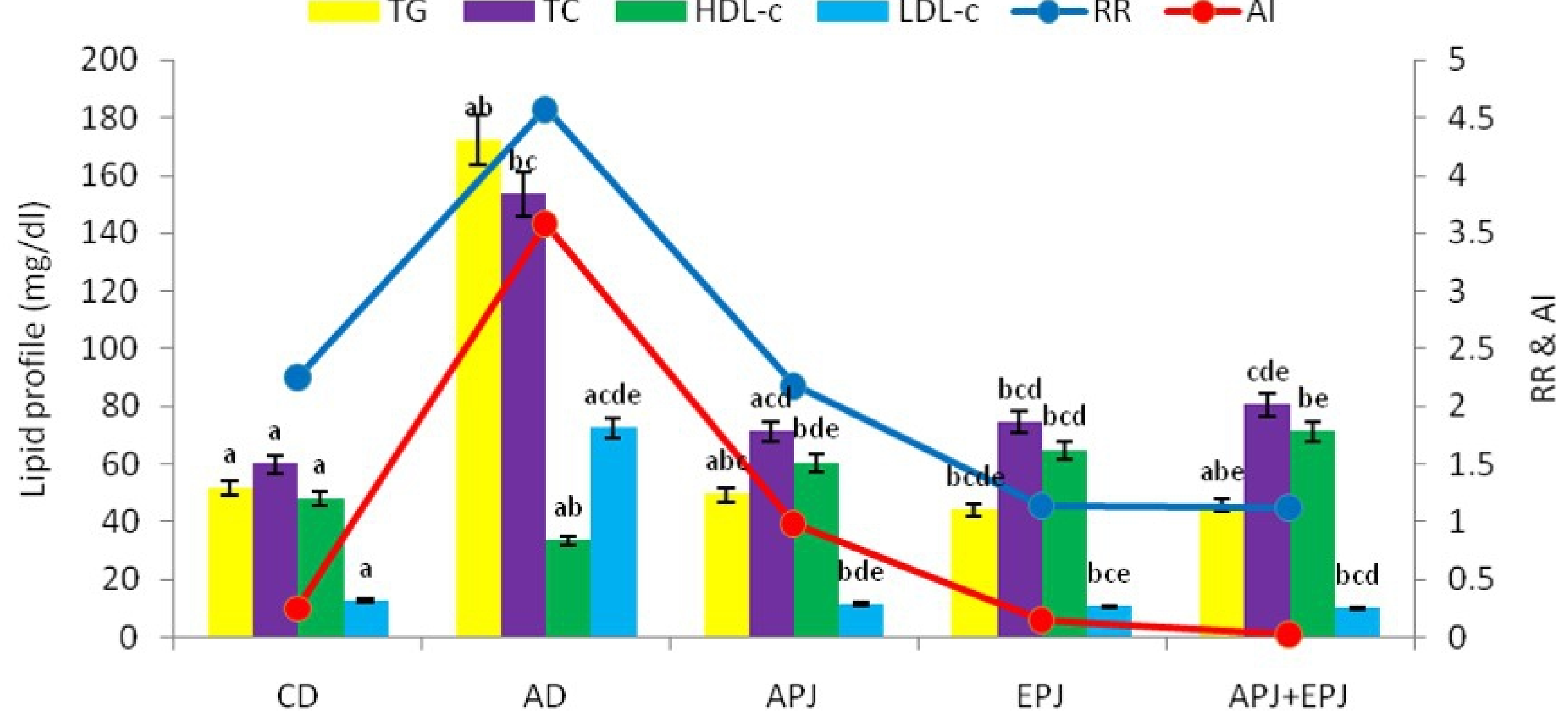

Treatment

Fig.2. Lipid profile in high-fat diet fed mice supplemented with 


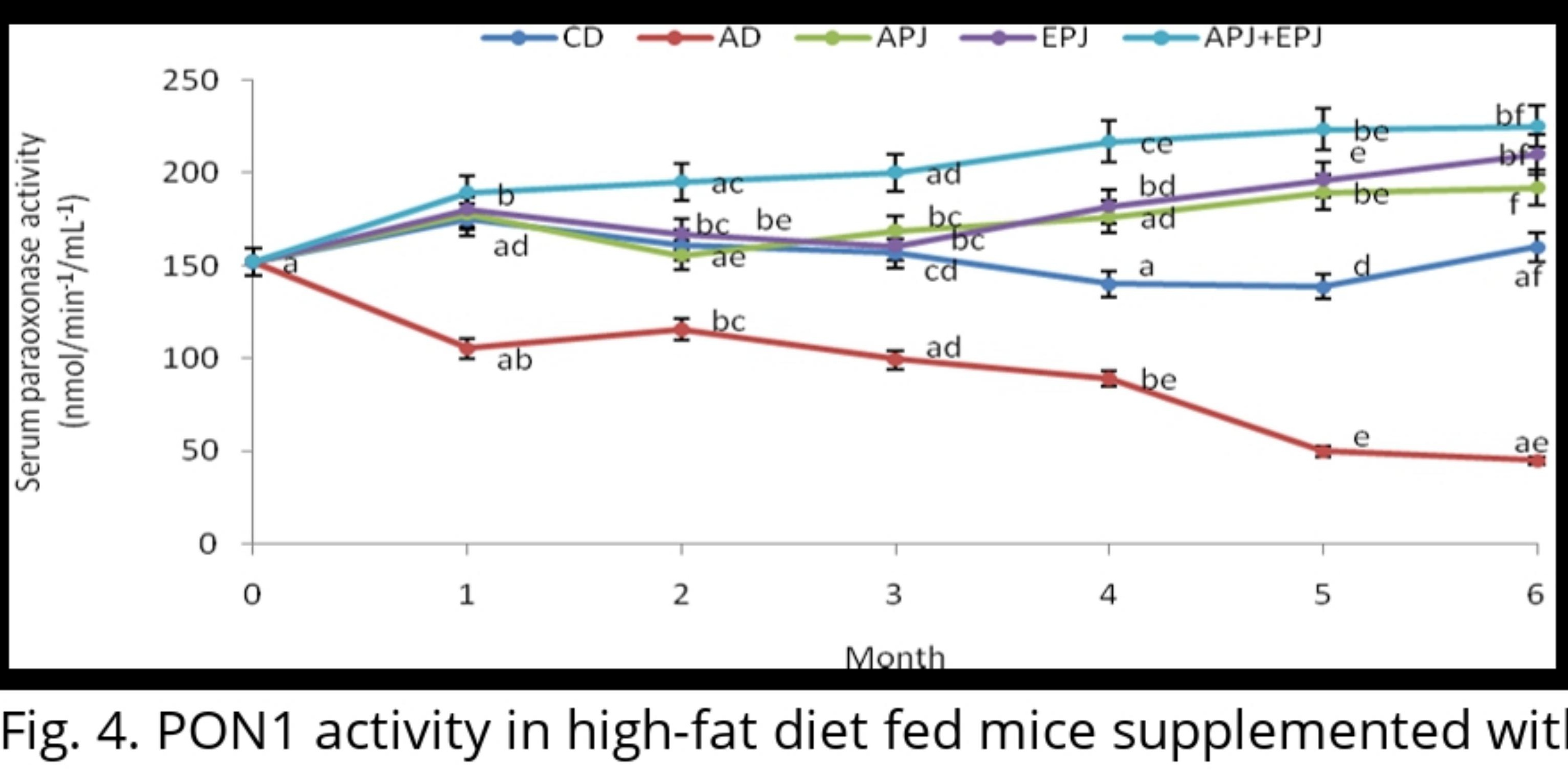




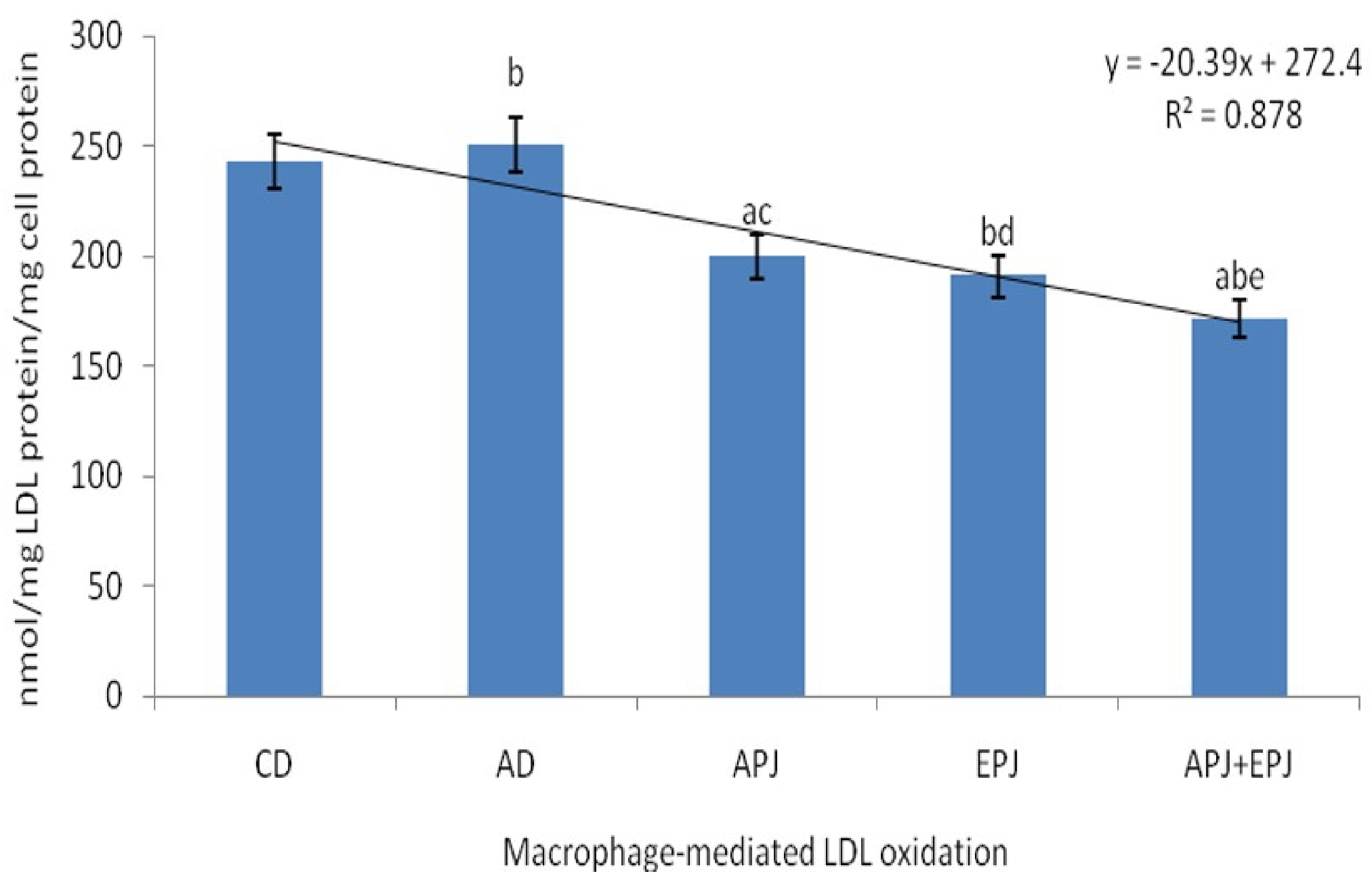

Fig. 5. Macrophage mediated LDL oxidation in high-fat diet fe 


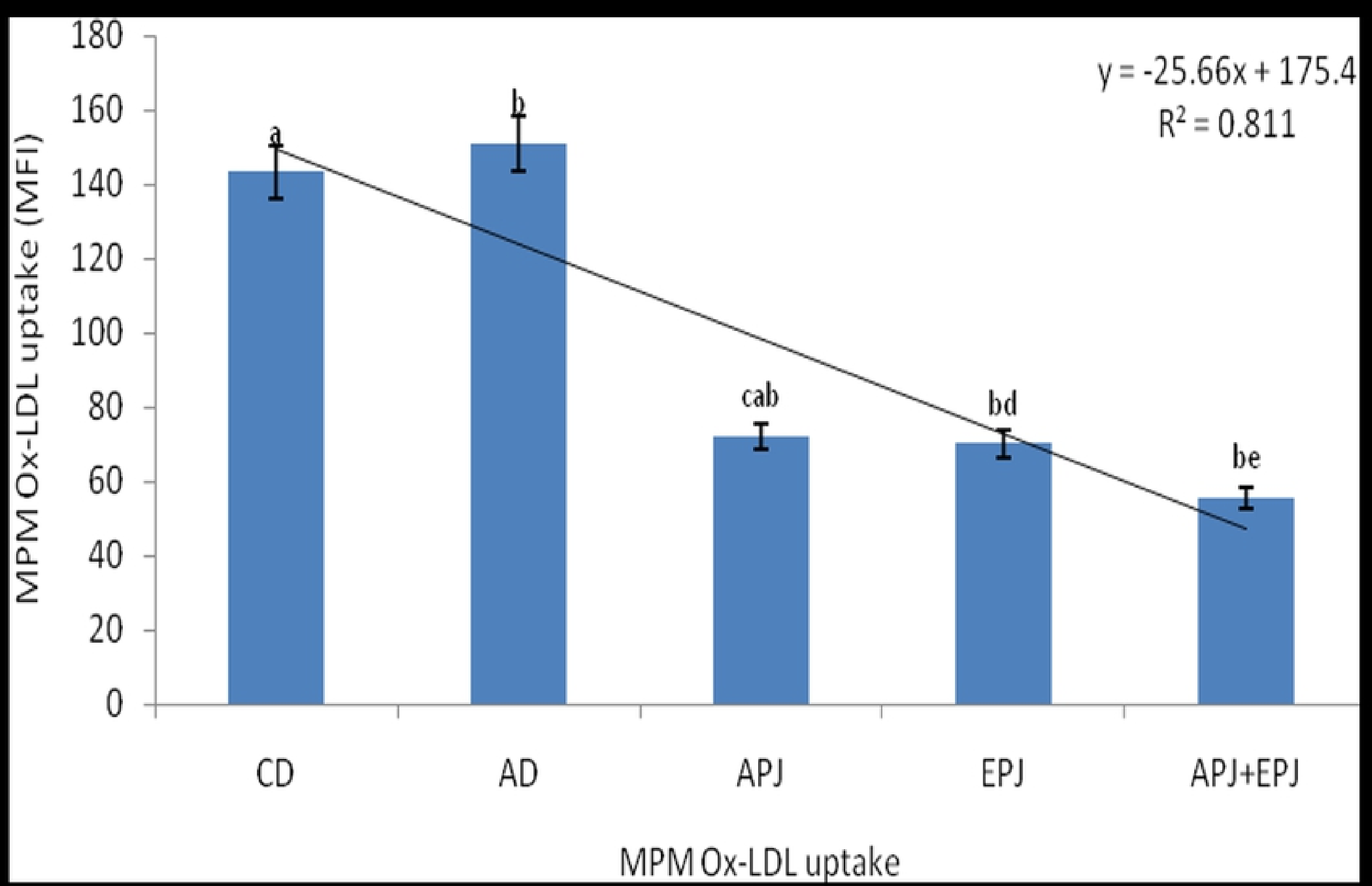

Fig. 6. Macrophage mediated Ox-LDL uptake in high-fat diet fed 


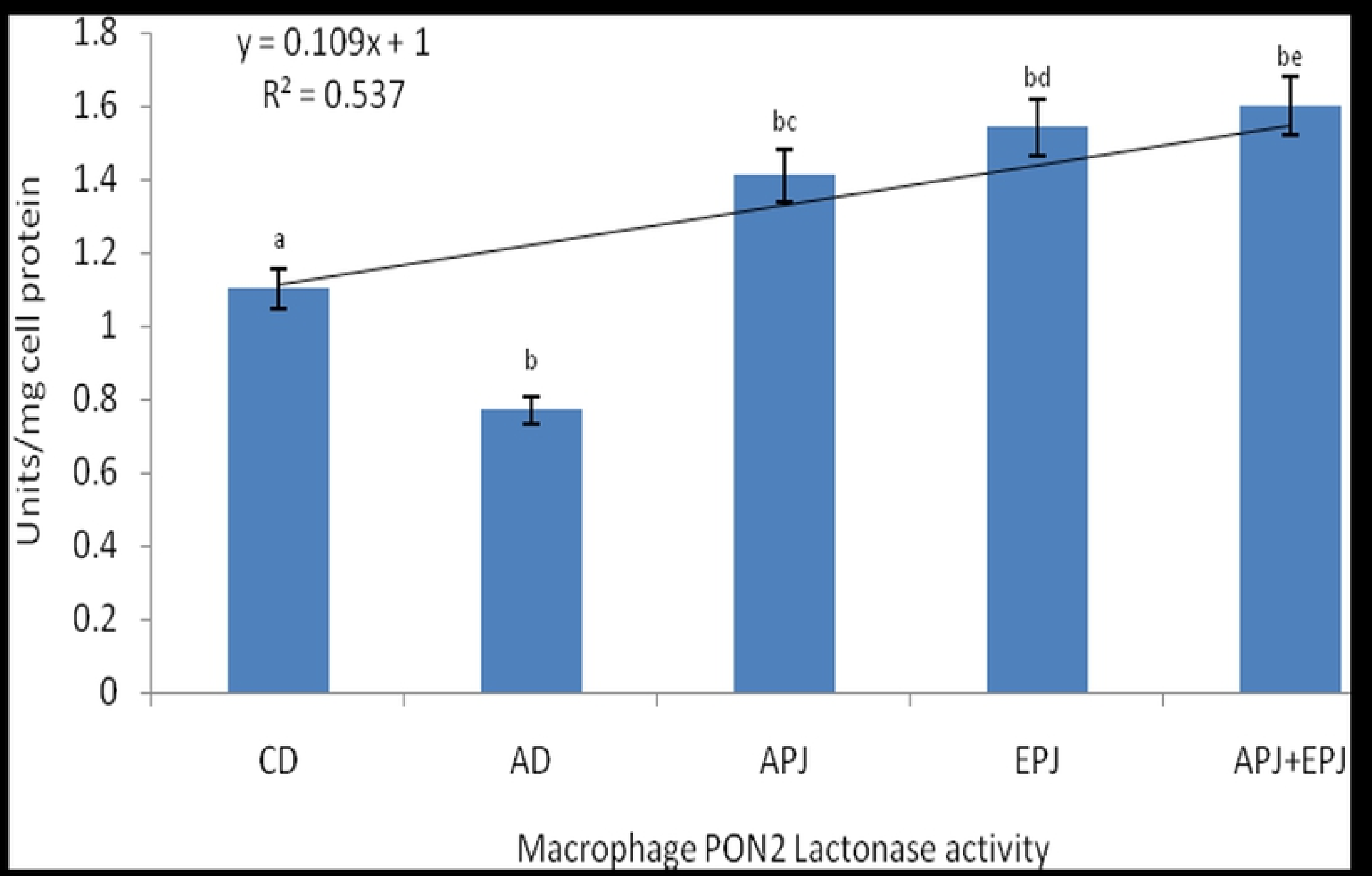

Fig. 7. Macrophage PON2 activity in high-fat diet fed mice supple 